\title{
RELATIONS AMONG HOMOTOPY OPERATIONS FOR SIMPLICIAL COMMUTATIVE ALGEBRAS
}

\author{
PAUL G. GOERSS AND THOMAS J. LADA
}

(Communicated by Thomas Goodwillie)

\begin{abstract}
The homotopy groups of a simplicial commutative algebra over the field with two elements support natural operations. Here we write the relations among these operations in an admissible form.
\end{abstract}

Let $A$ be a simplicial commutative algebra over the field $\mathbb{F}_{2}$. Then $A$ is, among other things, a simplicial set and, as such, has homotopy groups. In fact,

$$
\pi_{n} A \cong H_{n}(A, \partial)
$$

where $\partial=\sum d_{i}: A_{n} \rightarrow A_{n-1}$ is the sum (or alternating sum) of the face operators. By the Eilenberg-Zilber Theorem, $\pi_{*} A$ is a graded commutative $\mathbb{F}_{2}$ algebra. Cartan [2], Bousfield [1], and Dwyer [3] have pointed out the existence of natural operations on these homotopy groups. Indeed, Dwyer proved the following theorem.

Theorem 1. Let $A$ be a simplicial commutative $\mathbb{F}_{2}$ algebra. Then there are natural operations

$$
\delta_{i}: \pi_{n} A \rightarrow \pi_{n+i} A, \quad 2 \leq i \leq n,
$$

so that

(1) $\delta_{i}(x+y)=\delta_{i}(x)+\delta_{i}(y)$ if $i<n$ and $\delta_{n}(x+y)=\delta_{n}(x)+\delta_{n}(y)+x y$;

(2) $\delta_{i}(x y)=0$ unless $x \in \pi_{0} A$ or $y \in \pi_{0} A$. If $x \in \pi_{0} A$, then $\delta_{i}(x y)=$ $x^{2} \delta_{i}(y)$; if $y \in \pi_{0} A$, then $\delta_{i}(x y)=\delta_{i}(x) y^{2}$;

(3) if $j<2 i$, then there is a relation

$$
\delta_{j} \delta_{i}(x)=\sum_{(j+1) / 2 \leq k<i}\left(\begin{array}{c}
i-j+k-1 \\
i-k
\end{array}\right) \delta_{i+j-k} \delta_{k}(x) .
$$

Dwyer further points out that if $x \in \pi_{n} A, n \geq 1$, then $x^{2}=0$.

Received by the editors October 12, 1993 and, in revised form, January 20,1994; the second author presented the contents of this paper at the 92nd Annual Meeting of the AMS, January 7-11, 1986, New Orleans, LA.

1991 Mathematics Subject Classification. Primary 18G30, 55S99.

Key words and phrases. Simplicial commutative algebras, homotopy operations.

(C) 1995 American Mathematical Society 
The purpose of this note is to discuss the relations of Theorem 1, part (3). It is clear that any composition $\delta_{j} \delta_{i}$ can be rewritten as a linear combination of the compositions $\delta_{q} \delta_{p}$ which are admissible in the sense that $q \geq 2 p$. However, the relations as written do not immediately return such a sum but have to be applied recursively. Our main point is to get a closed formula, which is supplied in the next result.

Proposition 2. Let $A$ be a simplicial commutative $\mathbb{F}_{2}$ algebra and $x \in \pi_{n} A$. Then if $j<2 i$ there is a relation

$$
\delta_{j} \delta_{i}(x)=\sum_{(j+1) / 2 \leq k \leq(i+j) / 3}\left(\begin{array}{c}
i-j+k-1 \\
i-k
\end{array}\right) \delta_{i+j-k} \delta_{k}(x) .
$$

Notice that the binomial coefficients haven't changed (an amusing curiosity) but the limits on $k$ have; indeed, since $k \leq \frac{i+j}{3}$ one has $i+j-k \geq 2 k$ and the relations now return a sum of admissible compositions.

This result has been known to the second author for some time (see [5]) and was proved independently, but not published, by the first author for the work in [4].

The motivation for studying these relations was this: in the work of the first author on the cohomology of commutative $\mathbb{F}_{2}$ algebras [4], it is fairly simple to construct certain primary cohomology operations analogous to Steenrod operations. Then one wants to know the "Adem relations" among them. These are obtained by a "Koszul duality" argument (cf. [6]) once one has the relations among the operations $\delta_{i}$ in closed form, as in Proposition 2. And, in fact, it was low-degree calculations in the cohomology of commutative $\mathbb{F}_{2}$ algebras that suggested the truth of Proposition 2.

The proof of Proposition 2 is modeled on one of the standard ways cf producing the Adem relations in the Steenrod algebra. If $\mathscr{A}$ is the Steenrod algebra at the prime 2 , then there is a derivation $\partial: \mathscr{A} \rightarrow \mathscr{A}$ defined by

$$
\partial\left(\mathbf{S q}^{i}\right)=\mathrm{Sq}^{i-1}
$$

The Adem relations, then, are consequences of the existence of this derivation and the relations

$$
\mathrm{Sq}^{2 n-1} \mathrm{Sq}^{n}=0, \quad n \geq 1 \text {. }
$$

A similar argument will apply here.

We form an algebra analogous to the Steenrod algebra out of the operations $\delta_{i}$. Let $\mathscr{D}$ be the graded $\mathbb{F}_{2}$ algebra formed by taking the quotient of the tensor algebra on symbols $\delta_{i}$ of degree $i, i \geq 2$, by the ideal generated by the relations, for $j<2 i$

$$
R(j, i)=\sum_{(j+1) / 2 \leq k \leq i}\left(\begin{array}{c}
i-j+k-1 \\
i-k
\end{array}\right) \delta_{i+j-k} \delta_{k} .
$$

These are the relations implicit in Theorem 1, part (3).

The algebra $\mathscr{D}$ comes equipped with a degree-raising derivation $\partial: \mathscr{D} \rightarrow \mathscr{D}$. In fact, $\partial$ is defined by the requirement that it be a derivation over $\mathbb{F}_{2}$ and the formula

$$
\partial\left(\delta_{i}\right)=\delta_{i+1} \text {. }
$$

That this function is well defined is implied by the following result. 
Lemma 3. We have that

$$
\partial R(j, i)=R(j+1, i)+R(j, i+1)
$$

where, if $j+1=2 i, R(j+1, i)=0$.

Proof. If $j+1<2 i$, this is a routine, if tedious, exercise in binomial coefficients. Because of the denominator in the limits of the summation, it is easiest to break the calculation into two cases: one where $j$ is even and one where $j$ is odd. We omit the details. If $j+1=2 i$, one simply checks that

$$
\begin{aligned}
R(2 i-1, i) & =\delta_{2 i-1} \delta_{i} \\
R(2 i-1, i+1) & =\delta_{2 i} \delta_{i}+\delta_{2 i-1} \delta_{i+1}
\end{aligned}
$$

as required.

We next restate Proposition 2 in a form suitable for induction.

Proposition 4. Let $t>0$ and $2 b-t \geq 2$. Then in the algebra $\mathscr{D}$

$$
\delta_{2 b-t} \delta_{b}=\sum_{t / 3 \leq s \leq(t-1) / 2}\left(\begin{array}{c}
t-s-1 \\
s
\end{array}\right) \delta_{2 b-t+s} \delta_{b-s} .
$$

Notice that Proposition 4 implies Proposition 2 by setting $b=i, t=2 i-j$, and $s=b-k=i-k$.

The rest of this note is devoted to the proof of Proposition 4. This is accomplished by induction on $t$. The only point obscuring the argument is that because of the 2 and 3 in the denominators of the summation limits in the formula, there are six cases, one for each congruence class of $t$ modulo 6 .

To begin with, note that if $t=1$ or $t=2$, the formula in Proposition 5 merely restates $R(2 b-1, b)$ or $R(2 b-2, b)$ respectively. The cases $t=3,4,5$ and 6 are covered using the derivation $\partial$ as follows.

The case $t=3$ : One has

$$
0=\partial\left(\delta_{2 a-1} \delta_{a}\right)=\delta_{2 a} \delta_{a}+\delta_{2 a-1} \delta_{a+1} .
$$

Setting $a=b-1$ yields the desired formula.

The case $t=4$ : One has

$$
0=\partial\left(\delta_{2 a-2} \delta_{a}\right)=\delta_{2 a-1} \delta_{a}+\delta_{2 a-2} \delta_{a+1}=\delta_{2 a-2} \delta_{a+1} .
$$

Setting $a=b-1$ yields the desired formula.

The case $t=5$ : One has, by the case $t=3$,

$$
\partial\left(\delta_{2 a-3} \delta_{a}\right)=\partial\left(\delta_{2 a-2} \delta_{a-1}\right)
$$

which yields

$$
\delta_{2 a-3} \delta_{a+1}=\delta_{2 a-1} \delta_{a-1} .
$$

Again setting $a=b-1$ yields the desired result.

The case $t=6$ is similar.

By now the pattern is clear. Fix $t \geq 7$. Apply $\partial$ to

$$
\delta_{2 b-t+2} \delta_{b}=\sum_{(t-2) / 3 \leq s \leq(t-3) / 2}\left(\begin{array}{c}
t-s-3 \\
s
\end{array}\right) \delta_{2 b-t+2+s} \delta_{b-s}
$$


and use the fact that

$$
\delta_{2 b-t+3} \delta_{b}=\sum_{(t-3) / 3 \leq s \leq(t-4) / 2}\left(\begin{array}{c}
t-s-4 \\
s
\end{array}\right) \delta_{2 b-t+3+s} \delta_{b-s}
$$

to obtain a formula

$$
\begin{aligned}
\delta_{2 b-t+2} \delta_{b+1}= & \sum_{(t-3) / 3 \leq s \leq(t-4) / 2}\left(\begin{array}{c}
t-s-4 \\
s
\end{array}\right) \delta_{2 b-t+3+s} \delta_{b-s} \\
& +\sum_{(t-2) / 3 \leq s \leq(t-3) / 2}\left(\begin{array}{c}
t-s-3 \\
s
\end{array}\right) \delta_{2 b-t+3+s} \delta_{b-s} \\
& +\sum_{(t-5) / 3 \leq s \leq(t-5) / 2}\left(\begin{array}{c}
t-s-4 \\
s+1
\end{array}\right) \delta_{2 b-t+3+s} \delta_{b-s} .
\end{aligned}
$$

The difficulty comes in simplifying this formula, mostly because of the endpoints of the three summations are all different. We break this into six cases, one for each congruence class of $t$ modulo 6 . We do one case; the other cases are similar. So assume that $t=6 a+2$ and $a \geq 1$. Then

$$
\begin{aligned}
\delta_{2 b-t+2} \delta_{b+1}= & \sum_{2 a \leq s \leq 3 a-1}\left(\begin{array}{c}
t-s-4 \\
s
\end{array}\right) \delta_{2 b-t+3+s} \delta_{b-s} \\
& +\sum_{2 a \leq s \leq 3 a-1}\left(\begin{array}{c}
t-s-3 \\
s
\end{array}\right) \delta_{2 b-t+3+s} \delta_{b-s} \\
& +\sum_{2 a-1 \leq s \leq 3 a-2}\left(\begin{array}{c}
t-s-4 \\
s+1
\end{array}\right) \delta_{2 b-t+3+s} \delta_{b-s}
\end{aligned}
$$

This can be rewritten as

$$
\begin{aligned}
\delta_{2 b-t+2} \delta_{b+1}= & \left(\begin{array}{c}
t-2 a-3 \\
2 a
\end{array}\right) \delta_{2 b-4 a} \delta_{b-2 a+1} \\
& +\sum_{2 a \leq s \leq 3 a-2}\left(\begin{array}{c}
t-s-2 \\
s+1
\end{array}\right) \delta_{2 b-t+s+3} \delta_{b-s} \\
& +\left(\begin{array}{c}
3 a-1 \\
3 a-2
\end{array}\right) \delta_{2 b-3 a} \delta_{b-3 a+1}
\end{aligned}
$$

Since, by the case $t=2, \delta_{2 b-4 a} \delta_{b-2 a+1}=0$ and $\left(\begin{array}{c}3 a+1 \\ 3 a\end{array}\right)=\left(\begin{array}{c}3 a-1 \\ 3 a-2\end{array}\right)$, we have

$$
\delta_{2 b-t+2} \delta_{b+1}=\sum_{2 a \leq s \leq 3 a-1}\left(\begin{array}{c}
t-s-2 \\
s+1
\end{array}\right) \delta_{2 b-t+s+3} \delta_{b-s}
$$

or

$$
\delta_{2 b-t} \delta_{b}=\sum_{2 a+1 \leq s \leq 3 a}\left(\begin{array}{c}
t-s-1 \\
s
\end{array}\right) \delta_{2 b-t+s} \delta_{b-s}
$$

as predicted. 


\section{REFERENCES}

1. A. K. Bousfield, Operations on derived functors of non-additive functors, manuscript, Brandeis University, 1967.

2. H. Cartan, Algèbres d'Eilenberg-Mac Lane et homotopie, Seminaire Henri Cartan, 1954-55.

3. W. G. Dwyer, Homotopy operations for simplicial commutative algebras, Trans. Amer. Math. Soc. 260 (1980), 421-435.

4. P. G. Goerss, On the André-Quillen cohomology of commutative $\mathbb{F}_{2}$-algebras, Astérique 186 (1990).

5. T. J. Lada, Homotopy operations for simplicial commutative algebras, Abstract Amer. Math. Soc. 7 (1986), 125.

6. S. B. Priddy, Koszul resolutions, Trans. Amer. Math. Soc. 152 (1970), 39-60.

Department of Mathematics, University of Washington, Seattle, Washington 98195 E-mail address: pgoerss@math. washington. edu

Department of Mathematics, North Carolina State University, Raleigh, North CarOLINA 27695

E-mail address: lada@math.ncsu.edu 